\title{
ALIMENTATION STABILISÉE HAUTE TENSION POUR PHOTOMULTIPLICATEURS
}

\author{
Par P. CAROUSSOS, \\ Institut d'Optique, Paris.
}

\begin{abstract}
Résumé. - On présente une alimentation stabilisée haute tension spécialement conçue pour l'alimentation de photomultiplicateurs. On décrit successivement, en insistant sur leurs caractéristiques, les trois éléments qui la composent, à savoir une alimentation basse tension avec dispositif électronique de protection, un convertisseur haute tension et un régulateur. Le débit maximum est de $2 \mathrm{~mA}$ et la régulation obtenue de $0,3 \%$ pour la tension de sortie maximum.
\end{abstract}

Abstract. - A high voltage power supply, especially suited for photomultipliers, is described. The three principal units are described in detail : a low voltage supply with electronic protection, a high voltage converter and a regulator.

The maximum current is $2 \mathrm{~mA}$ and the regulation is. $3 \times 10^{-4}$ for the maximum nutput voltage.

Le nombre des montages optiques qui utilisent des photomultiplicateurs étant de plus en plus important dans nos laboratoires, nous avons été conduit à étudier des alimentations $H$. T. stabilisées pour l'alimentation de ces photomultiplicateurs.

Nous allons décrire ici une alimentation dont le débit maximum est de $2 \mathrm{~mA}$, par conséquent largement suffisant dans tous les montages avec des photomultiplicateurs dont la consommation continue n'est guère supérieure à $1 \mathrm{~mA}$.

Cette alimentation H. T. stabilisée, dont le schéma est donné dans la figure 1, est constituée d'une alimentation B. T. avec dispositif électronique de protection, d'un convertisseur $H$. T. et d'un régulateur.

1. Alimentation B. T. - Cette alimentation, dont la tension de sortie de $-17,5$ volts est stabilisée à $1 \%$ pour un débit de $800 \mathrm{~mA}$, alimente à travers le régulateur le convertisseur $\mathrm{H}$. T.

Afin que la tension de sortie de l'alimentation B. T. soit indépendante des variations de température éventuelles, le coefficient thermique $\Delta V_{\mathrm{R}} / \Delta T$ de la tension de référence $V_{\mathrm{R}}$ (tension aux bornes

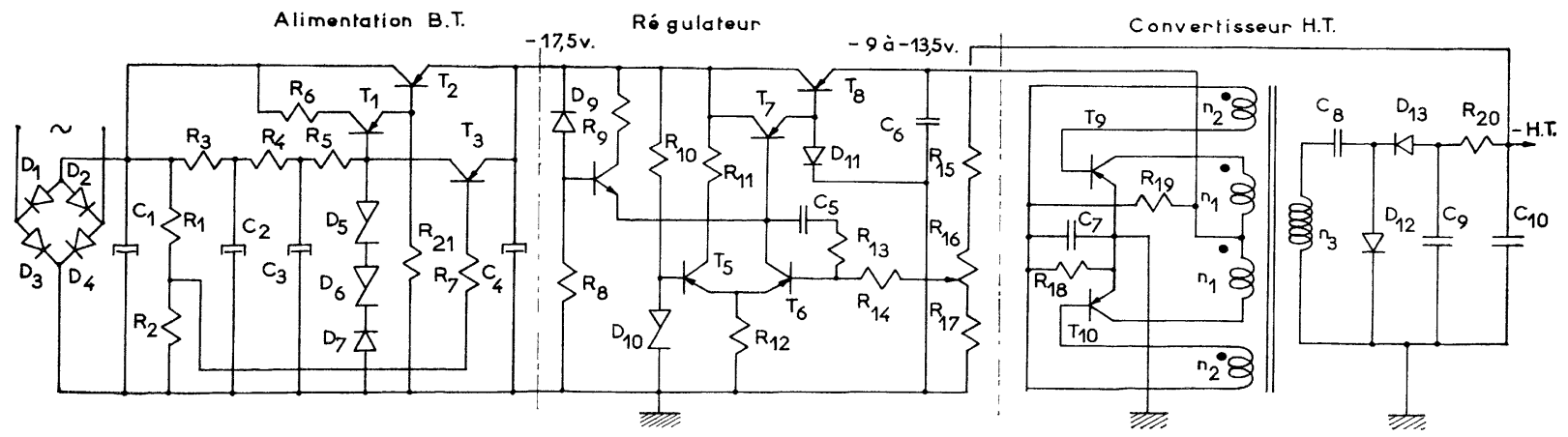

FIG. 1. -.. Alimentation haute tension stabilisée.

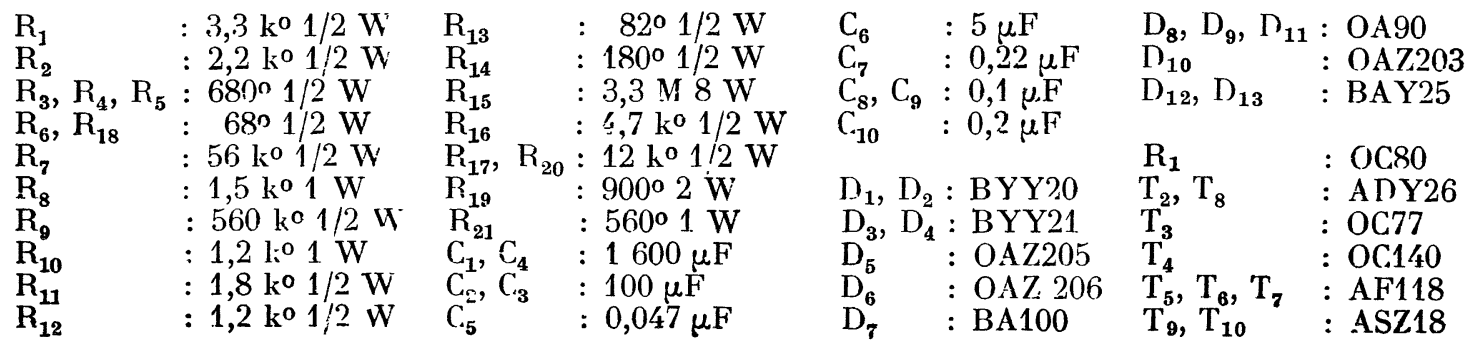


des $D_{5}, D_{6}$ et $\left.D_{7}\right)$ doit compenser le coefficient thermique négatif $\Delta V_{\mathrm{BE}} / \Delta T$ de la tension $V_{\mathrm{BE}}$ entre la base du transistor $T_{1}$ et l'émetteur du transistor $T_{2}$. Par conséquent, le coefficient $\Delta V_{\mathrm{R}} / \Delta T$ doit être positif et égal en valeur absolue au coefficient $\Delta V_{\mathrm{BE}} / \Delta T$. Le coefficient thermique des deux diodes Zener $D_{5}$ et $D_{6}$ choisies est positif mais de valeur absolue plus élevée qu'il n'est nécessaire. La compensation exacte est obtenue grâce au coefficient thermique négatif de la diode au silicium $D_{7}$.

Les courants de fuite des transistors $T_{1}$ et $T_{2}$ sont éliminés par la résistance $R_{21}$, qui permet également un bon fonctionnement pour de faibles courants de charge.

La protection contre les surcharges est obtenue par le transistor $T_{3}$ qui agit comme un interrupteur électronique. En fonctionnement normal le transistor $T_{3}$ est coupé : la tension de la base est plus positive que la tension à l'émetteur (la tension entre les résistances $R_{1}$ et $R_{2}$ est de l'ordre de -13 volts par rapport à la masse, tandis que la tension de sortie est de $-17,5$ volts). Si, par suite d'une surcharge, le courant à débiter est supérieur à 1 ampère, la tension de sortie tend vers zéro et le transistor $T_{3}$ commence à conduire. Le courant $I_{\mathrm{B}}$ à travers la résistance $R_{7}$ est suffisamment important pour que le transistor $T_{3}$ se sature. La tension $V_{\mathrm{CE}}$ de saturation étant pratiquement nulle, il en est de même pour la tension entre la base du transistor $T_{1}$ et l'émetteur du transistor $T_{2}$; par conséquent, celui-ci ne débite plus. Les essais effectués ont démontré l'efficacité de ce système de protection.

2. Le convertisseur H. T. - Le convertisseur transforme la tension continue fournie par le régulateur en une tension alternative rectangulaire aux bornes des enroulements $n_{1}$. La tension induite aux bornes de l'enroulement $n_{3}$ est proportionnelle au rapport de transformation $n_{3} / n_{1}$. Le transformateur du convertisseur possède un cycle d'hystérésis rectangulaire.

Le couple de transistors $T_{9}$ et $T_{10}$ agit comme un double inverseur. Quand le transistor $T_{9}$ conduit, le transistor $T_{10}$ est bloqué et inversement. Supposons que le transistor $T_{9}$ commence à conduire : la borne de l'enroulement $n_{1}$ connectée au collecteur du transistor $T_{9}$ devient de plus en plus positive. Cette tension positive croissante induit aux bornes des autres enroulements des tensions dont les amplitudes sont proportionnelles au rapport du nombre de spires, et dont les signes sont indiqués par les points. Par conséquent, la borne de l'enroulement $n_{2}$ connectée à la base du transistor $T_{9}$ devient négative et renforce le courant du collecteur du même transistor; par contre, la base du transitor $T_{10}$ devient plus positive et bloque davantage le transistor $T_{10}$. A un certain moment, le circuit magnétique du transformateur se sature et de ce fait les tensions induites s'annulent. La tension aux bornes de l'enroulement $n_{1}$ connecté à $T_{9}$ s'inverse, ce qui entraîne la conduction du transistor $T_{10}$ et le blocage complet du transistor $T_{9}$, et ainsi de suite.

Le circuit magnétique du transformateur du convertisseur est un ferroxcube $\mathrm{H}$ de la R. T. C. Afin de déterminer le nombre d'ampère-tours nécessaires pour le saturer, nous avons utilisé le montage suivant : Nous avons bobiné autour du circuit magnétique trois enroulements comportant chacun un nombre égal de spires $n=30$. On applique un courant continu variable aux bornes du premier enroulement, une tension sinusoïdale de 1 volt c. à. c. au deuxième enroulement et on connecte le troisième à un oscilloscope. Si, dans le premier enroulement, on augmente le courant continu à partir de zéro, l'amplitude du signal (d'un volt c. à. c. sur l'écran de l'oscilloscope) reste d'abord constante. Si l'on continue à augmenter le courant, l'amplitude du signal diminue, car le circuit magnétique du transformateur commence à se saturer. Dans notre cas, le ferroxcube se sature pour un courant de $270 \mathrm{~mA}$. Par conséquent, le nombre d'ampère-tours de saturation est : $n I=8,1$ At. Ainsi, si on adopte pour chaque transistor un courant $I_{\mathrm{C}}$ de saturation de $900 \mathrm{~mA}$, les deux enroulements $n_{1}$ doivent comporter chacun neuf spires. Si, d'autre part, on considère que la tension continue fournie par le régulateur au convertisseur est de 12 volts (ce qui donne entre les deux collecteurs des transistors $T_{\mathbf{9}}$ et $T_{\mathbf{1 0}}$ un signal rectangulaire d'une amplitude minimum de 24 volts), et que la tension à vide du secondaire H. T. doit être de 1000 volts, le nombre de spires $n_{3}$ de ce dernier enroulement doit être $18 \times 1000 / 24=750$ spires. Étant donné que le secondaire doit fournir un certain débit, nous avons adopté pour $n_{3}=850$ spires. Enfin, chaque enroulement $n_{2}$ est constitué d'une spire.

L'ensemble $C_{8}, C_{9}, D_{12}$ et $D_{13}$ forme un doubleur de tension. L'ensemble $R_{20}$ et $C_{10}$ est un filtre passebas. Les résistances $R_{18}$ et $R_{19}$ fournissent une polarisation négative aux bases des transistors $T_{9}$ et $T_{10}$ pour assurer l'autodémarrage de l'oscillateur lors de la mise sous tension. Le condensateur $C_{7}$ supprime les oscillations transitoires qui risqueraient de détériorer les transistors.

3. Le régulateur. - La tension de sortie du convertisseur étant proportionnelle à la tension continue fournie à l'oscillateur par le régulateur, la stabilisation du convertisseur est obtenue par la régulation de cette tension continue. Suivant la tension de sortie du convertisseur, et pour un débit de 0 à $2 \mathrm{~mA}$, la tension continue varie de -9 à $-13 \mathrm{~V}$.

Une partie de la haute tension, obtenue par le diviseur $R_{15}, R_{16}, R_{17}$, est appliquée à l'une des entrées de l'amplificateur de différence (transistors $T_{5}$ et $T_{6}$ ), et elle est comparée à la tension de référence appliquée à la deuxième entrée de cet 
amplificateur. La tension de référence est obtenue par la diode Zener $D_{10}$, qui doit posséder un coefficient thermique aussi faible que possible.

Pour avoir une bonne régulation, l'amplificateur de différence doit avoir un gain en tension élevé. Pour cela, la résistance de charge du collecteur du transistor $T_{6}$ doit être grande, ce qui nécessite la connection de cette résistance à une source de tension auxiliaire plus négative que la tension existante à l'entrée du régulateur, afin que le courant du collecteur ne soit pas trop faible. Pour éviter l'emploi de cette source de tension auxiliaire, nous avons remplacé la charge du collecteur par l'ensemble $D_{9}, R_{8}, R_{9}, T_{4}$, qui n'est autre qu'une source de courant $\left(I_{\mathrm{CA}} \simeq 1,4 \mathrm{~mA}\right)$ et qui présente une résistance dynamique élevée (de l'ordre de $60 \mathrm{k} \Omega$ ).

La diode $D_{11}$ permet l'évacuation des porteurs minoritaires du collecteur du transistor $T_{8}$ qui émigrent vers sa base (courant $I_{\mathrm{CBO}}$ ), et compense le courant $I_{\mathrm{CBO}}$ du transistor $T_{7}$. Par conséquent, la diode $D_{11}$ choisie doit avoir un courant inverse, supérieur ou égal à la somme des courants $I_{\mathrm{CBO}\left(\mathrm{T}_{8}\right)}+I_{\mathrm{CEO}(\overline{7})}\left(I_{\mathrm{inv}} \simeq 660 \mu \mathrm{A}\right.$ à $\left.25^{\circ} \mathrm{C}\right)$. Étant donné que les courants $I_{\mathrm{CBO}}$ et $I_{\mathrm{CEO}}$ augmentent avec la température, la diode $D_{11}$ est fixée sur le radiateur du transistor $T_{8}$, pour que le courant $I_{\text {inv }}$ de la diode augmente de la même façon. L'ensemble $C_{5}, R_{13}$ diminue les oscillations transitoires par rétroaction négative.

4. Performances. - L'alimentation stabilisée H. T. décrite présente les caractéristiques suivantes : Si la valeur de la résistance $R_{17}$ est de $12 \mathrm{k} \Omega$, la tension de sortie de l'alimentation $H$. T. varie, pour les deux positions extrêmes du potentiomètre $R_{16}$, de $1250 \mathrm{~V}$ à $1780 \mathrm{~V}$ avec un débit de $2 \mathrm{~mA}$. L'ondulation résiduelle est inférieure à $10 \mathrm{mV}$ c. à. c. A 1780 volts, pour une variation du débit de 1,07 mA, la variation de la $H$. T. est de $0,468 \mathrm{~V}$. Par conséquent, la régulation est de $0,468 / 1780 \simeq 0,263 \%$ et la résistance dynamique est de $0,468 \mathrm{~V} / 1,07 \mathrm{~mA}=437 \mathrm{ohms}$. Il est possible d'obtenir une régulation encore meilleure en utilisant deux amplificateurs de différence en cascade.

Sans que la miniaturisation ait été poussée à l'extrême, l'ensemble électronique décrit est contenu dans une boîte de dimensions $13 \times 17,5 \times 15 \mathrm{~cm}^{3}$.

Manuscrit reçu le 12 juillet 1966. 